\title{
Measurement of consummatory behavior in honey bees
}

\author{
J. E. SIGURDSON \\ Békésy Laboratory of Neurobiology, University of Hawaii, Honolulu, Hawaii 96822
}

\begin{abstract}
Consummatory behavior in honey bees can be measured by recording contact of the proboscis with sucrose solution in the food cup of an automatic feeder, and the behavior can be instrumentalized by arranging for contact of the proboscis with the food cup to activate the feeder. Illustrative free-operant and discrete-trials data are presented.
\end{abstract}

In a recent paper on techniques for the study of appetitive conditioning in honey bees (Sigurdson, 1981), I described a contact-detecting circuit used to measure the approach of a bee to a feeding place. With the same circuit used to detect contact of the proboscis with sucrose solution in a food cup or with the food cup itself, consummatory responding can be measured and conditioned in honey bees in much the same way as it has been in a diverse array of other animals, including goldfish (Holmes \& Bitterman, 1969), painted turtles (Pert \& Bitterman, 1969), and Mayan octopuses (Henderson, Woodard, \& Bitterman, 1975).

\section{APPARATUS}

The apparatus is diagrammed in Figure 1. Its central feature is a food cup ( $2.3 \mathrm{~mm}$ in diameter) to which sucrose solution can be delivered by a syringe pump. The cup, and the stem through which food is delivered to the cup, are milled from a No. 22 Luer-Lok needle. The sensory lead of a capacitive transducer already described (Sigurdson, 1981) is attached to the stem, and all metal objects within $20 \mathrm{~cm}$ of the fluid system are grounded. The outside of the cup is coated with an insulating material, but when the proboscis of the bee makes contact with the inside surface of the cup, or with the sucrose solution that it may contain, the capacitance of the system increases, resulting in the operation of a response relay. The Plexiglas chamber to which the bee comes from the hive is, like chambers previously described, connected to the outside by a short tunnel. The tunnel is equipped with photodetectors and doors that can be used to control the entrance and departure of the animal if temporary confinement is required on any given visit. Set into the floor of the chamber is a covered reservoir of sucrose solution (a free-feeder) that can be uncovered and illuminated

This paper is adapted from a doctoral dissertation submitted to the University of Hawaii. The work was done under the direction of M. E. Bitterman with the support of Grant BNS 7905876 from the National Science Foundation. Requests for reprints should be addressed to the Békésy Laboratory of Neurobiology, 1993 East-West Road, Honolulu, Hawaii 96822.
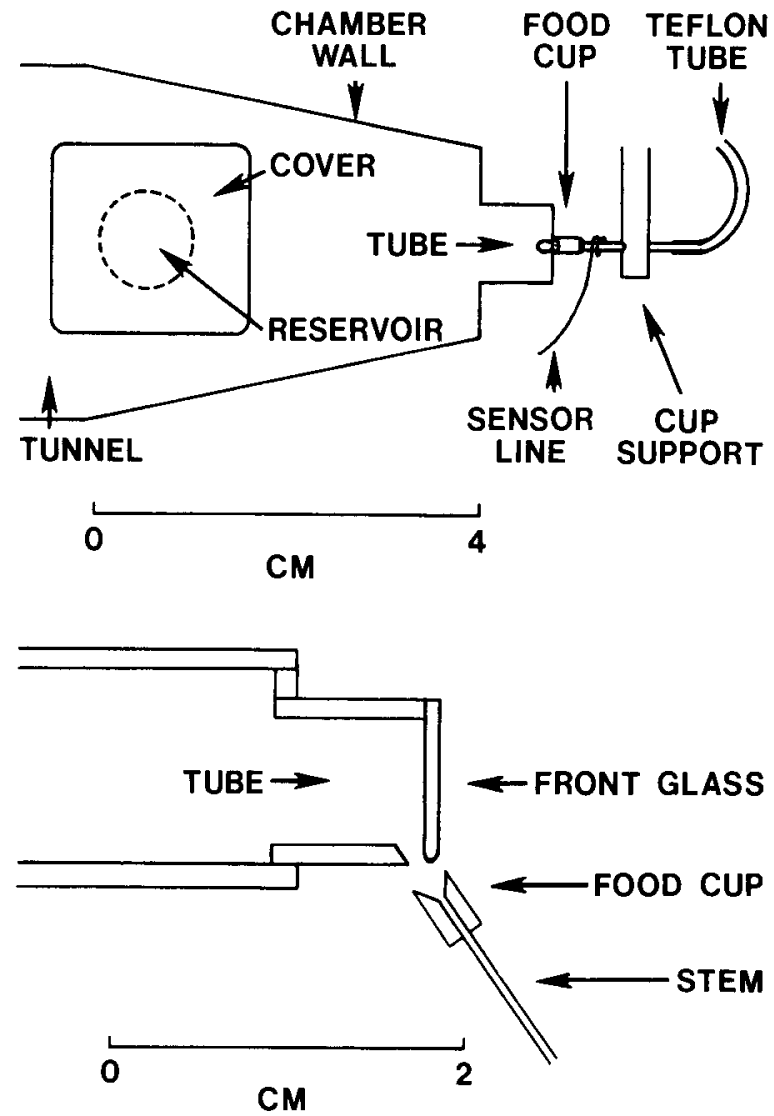

Figure 1. (Upper portion) Plexiglas chamber of the apparatus. (Lower portion) Cross-section of the glass tube and food cup.

automatically, permitting the animal to feed to repletion before leaving the chamber when a full crop load is not provided by the training schedule employed. The front wall of the glass tube that gives access to the food cup is of clear glass, and through it the colored lights that serve as conditioned stimuli are visible.

\section{ILLUSTRATIVE DATA}

\section{Free-Operant Training}

Instead of measuring the duration of contact between proboscis and feeder, I measured discrete responses by 
introducing what was in effect an instrumental contingency. Each contact programmed the delivery of 2 microliters of sucrose solution ( $35 \%$ by weight), continuous contact with the cup being required to keep the pump-timer running. When, however, the delivery of the programmed amount of reinforcement was complete, the animal was required to break the contact (which tended to happen spontaneously anyway) and to make contact again in order to activate the feeder once more. From this continuous reinforcement (CRF) training, it was easy to go to ratio and interval schedules.

Shown in Figure 2 is the cumulative record of a single bee trained on a CRF schedule and then given an increasing fixed-ratio (FR) requirement. The animal was introduced to the chamber in the usual way (Sigurdson, 1981) and fed to repletion from a large drop of sucrose solution. On its first spontaneous return to the apparatus, the bee was confined until it discovered the food cup, which was elevated to the level of the tube floor and then gradually lowered $2 \mathrm{~mm}$ to its final position as the bee drank its fill with no interruption (the pump running continuously). On the next visit, the CRF schedule was

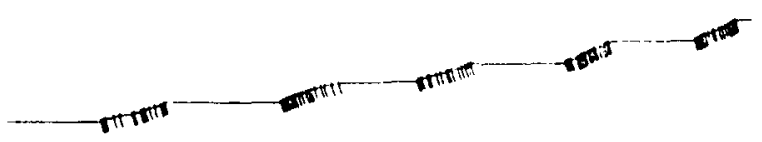

VISITS 1.5 [CRF]

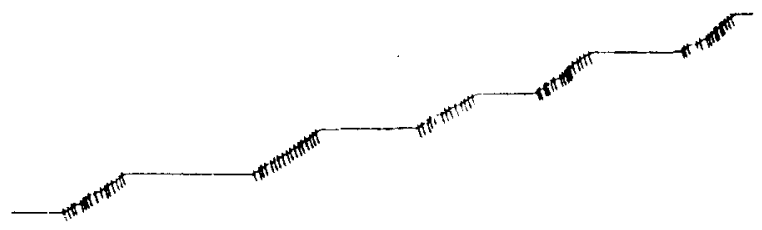

VISITS 6-10 |FR-2]

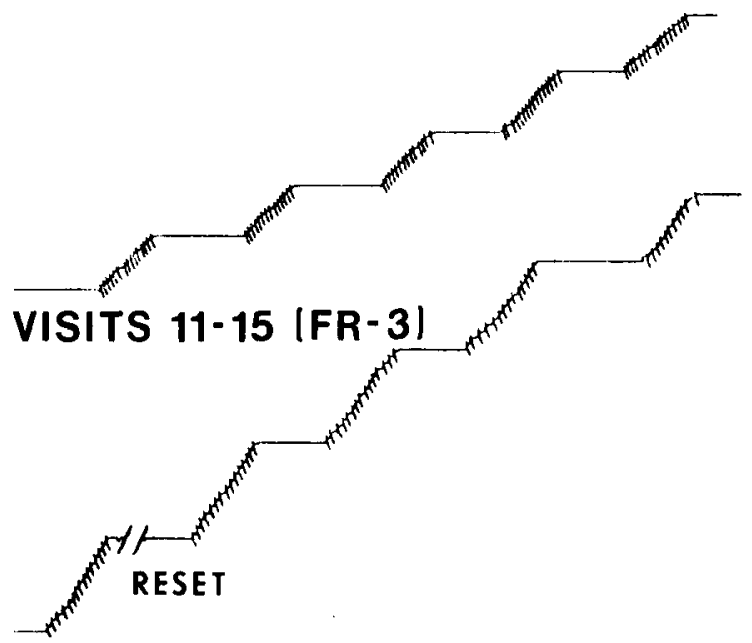

\section{VISITS 16-20 (FR-5)}

Figure 2. Acquisition of consummatory responding on a CRF schedule and subsequent FR schedules.

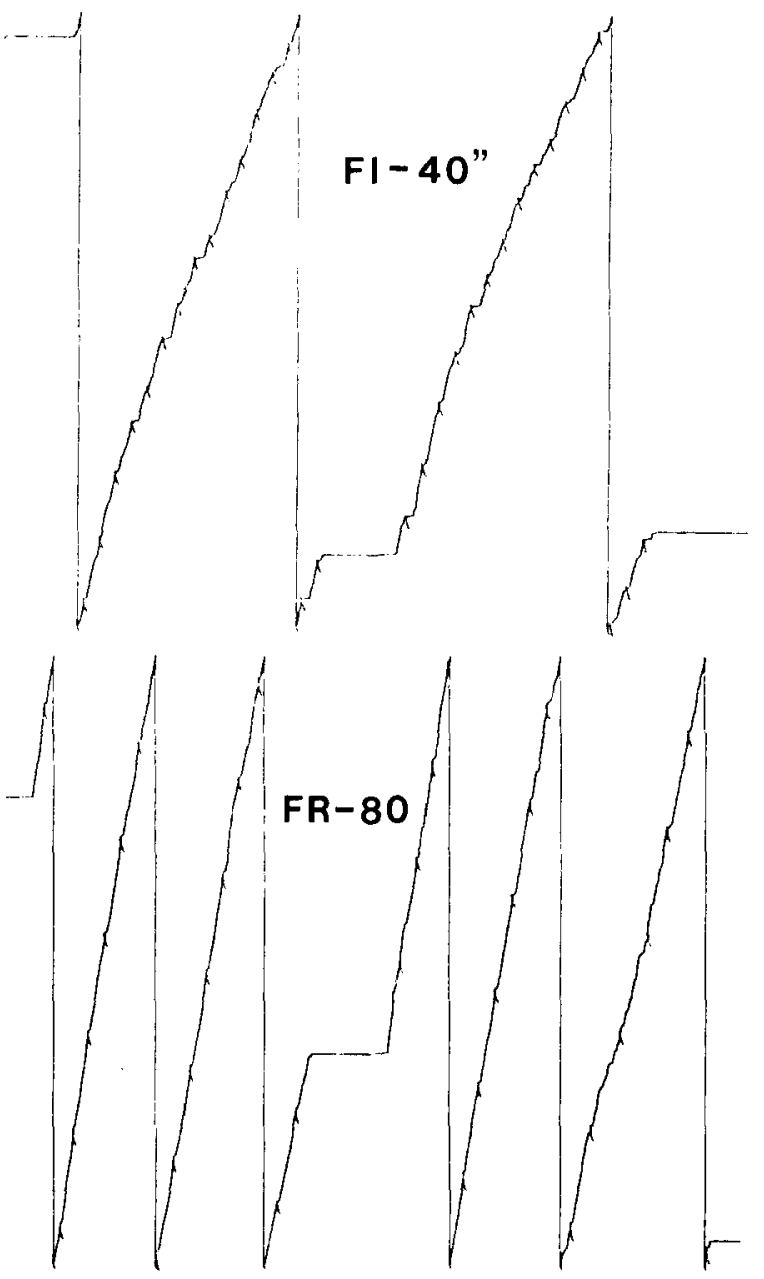

Figure 3. Consummatory responding on FI 40-sec and FR 80 schedules of reinforcement.

introduced and the recording began. The tracing is to be interpreted in the conventional way, the pen stepping upward with each response and diagonally with each reinforcement. The clusters of responses and reinforcements show performance in successive visits to the chamber, whereas plateaus between clusters mark returns to the hive. In this experiment, the door to the outside remained open, and the animal was free to come and go as it liked. After five CRF visits, a ratio requirement was introduced: FR 2 in Visits $6-10, F R 3$ in Visits 11-15, and then FR 5, to which the animal adjusted rapidly. Under these conditions, the animals respond at high rates on relatively thin schedules of reinforcement. Shown in Figure 3 are some samples of fixed-interval (FI) 40 -sec and FR 80 performance.

\section{Discrete-Trials Discrimination}

The temporary-confinement technique may be used for discrete-trials experiments with proboscis contact as the response in the same way as in previous work with the dipping and approach responses (Sigurdson, 1981). Illustrated here is the use of the method for discriminative training by the discrete-trials/FI procedure of 


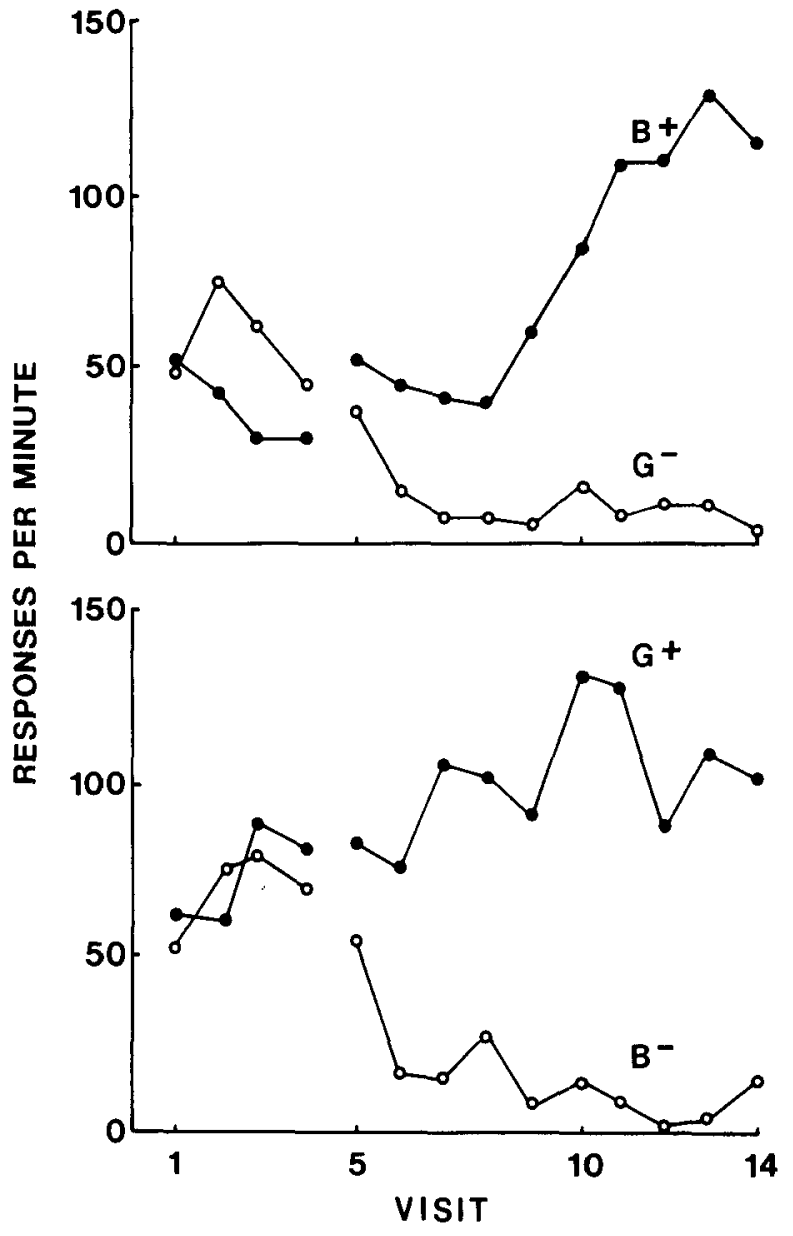

Figure 4. Mean rate of response of two bees on nondifferential (Visits 14) and discrimination (Visits 5-14) training.

Woodard and Bitterman (1974). Each bee was introduced to the apparatus in the manner described above. On its second spontaneous visit, the bee was automatically admitted and confined to the chamber, and after a delay for adaptation, the white stimulus lamp was illuminated and response to the food cup was reinforced with 1.5 microliters of $35 \%$ sucrose solution on a CRF schedule. As before, continuous contact with the food cup was required to keep the pump running to the completion of each reinforcement cycle. After 20 reinforcements, the white lamp was turned off, and the bee was given access to the free-feeder, from which it filled its crop before departing for the hive. This schedule remained in effect for several visits, after which it was modified. Now, on each visit there were four discrete presentations of the white light separated by intervals in darkness whose duration was controlled by a $5-\mathrm{sec}$ timer that was reset by response. Initially, each presentation of the white light signaled the immediate availability of five response-contingent reinforcements, but then an FI between the start of the trial and the availability of reinforcement was introduced and was gradually increased to $8 \mathrm{sec}$. The number of food-cup contacts within the interval was recorded and provided the basic measure of performance. In the final stages of pretraining, there were 108 -sec trials per visit, each trial terminating with two response-contingent reinforcements and each visit terminating as before, with access to the free-feeder.

When responding had stabilized, the white-light trials were replaced by trials with blue (B) and green (G) lights, 10 each in Gellermann orders. Both colors were reinforced for four visits, after which the discrimination training was begun, $\mathrm{B}+\mathrm{G}-$ for one animal, $\mathrm{G}+\mathrm{B}-$ for a second. The procedure for the positive color was the same as before, but the negative color was simply turned off after $8 \mathrm{sec}$ without reinforcement. This schedule remained in effect for 10 visits. In Figure 4, the performance of the two bees is plotted in terms of mean rate of responses to each color on each visit. The $\mathrm{G}+\mathrm{B}$ animal showed no color preference in the nondifferential training and rapid discrimination in the subsequent differential training. The $B+G-$ animal showed some preference for green in the nondifferential training and somewhat less rapid discrimination, although by the last four visits it was responding to the positive and negative colors at rates differing by an order of magnitude. There was evidence in the performance of both animals of the positive contrast found earlier with the dipping response (Sigurdson, 1981).

This consummatory technique has application to a wide range of analytical experiments on motivation and learning in honey bees.

\section{REFERENCES}

Henderson, T. B., Woodard, W. T., \& Bittenman, M. E Measurement of consummatory behavior in octopuses Behavior Research Methods \& Instrumentation, 1975, 7 265-266.

Holmes, N. K., \& Bitterman, M. E. Measurement o consummatory behavior in the fish. Journal of the Experimenta Analysis of Behavior, 1969, 12, 39-41.

Pert, A., \& Bitterman, M. E. A technique for the study o consummatory behavior and instrumental learning in the turtle American Psychologist, 1969, 24, 258-261.

Sigurdson, J. E. Automated discrete-trials techniques of appeti tive conditioning in honey bees. Behavior Research Methods d Instrumentation, 1981, 13, 1-10.

Woodard, W. T., \& BitTenman, M. E. A discrete-trials/fixed interval method of discrimination training. Behavior Researc/ Methods \& Instrumentation, 1974, 6, 389-392.

(Received for publication June 3, 1981; accepted June 26, 1981.) 